\title{
Knowledge Enhanced E-government Portal
}

\author{
Jan Paralic ${ }^{1}$, Tomas Sabol${ }^{2}$, and Marian Mach ${ }^{1}$ \\ ${ }^{1}$ Dept. of Cybernetics and AI, Technical University of Kosice, Letna 9, \\ 04200 Kosice, Slovakia \\ Jan.Paralic@tuke.sk \\ ${ }^{2}$ Faculty of Economics, Technical University of Kosice, B. Nemcovej 32, \\ 04200 Kosice, Slovakia \\ Tomas.Sabol@tuke.sk
}

\begin{abstract}
There is a growing number of e-Government portals and solutions available today. But what the users lack in particular is a customised assistance - help that meets the individual situation and competence [13]. In this paper, a system called Webocrat will be presented as an attempt to shift e-Government portals toward this direction, providing knowledge management strategy as its basis [11]. The Webocrat system applies a knowledge-based approach [5]. Information of all kinds produced by various modules is linked to a shared ontology representing an application domain. Such ontology serves as a means for structuring and organizing available information resulting in improved search capability and contents presentation.
\end{abstract}

\section{Introduction}

Knowledge can be simply defined as actionable information [12]. That means that (only) relevant information available in the right place, at the right time, in the right context, and in the right way can be considered as knowledge.

The knowledge life cycle defined in [9] hinges on the distinction between tacit and explicit knowledge. Explicit knowledge is a formal one and can be found in documents of an organization: reports, manuals, correspondence (internal and external), patents, pictures, tables (e.g. Excel sheets), images, video and sound recordings, software etc. Tacit knowledge is personal knowledge given by individual experience (and hidden in peoples' minds) [12].

A considerable pmount of explicit knowledge is scattered throughout various documents within public and governmental organizations and people minds working there. In many cases the possibility to efficiently access (retrieve) and reuse this knowledge is limited [3]. As a result of this, most knowledge is not sufficiently exploited, shared and supsequently forgotten in relatively short time after it has been introduced to, invented/discovered within the organization. Therefore, in the approaching information society, it is vitally important for knowledge-intensive organizations as public and governmental institutions to make the best use of information gathered from various information resources inside the organizations and from exter-

The original version of this chapter was revised: The copyright line was incorrect. This has been corrected. The Erratum to this chapter is available at DOI: 10.1007/978-3-540-44836-5_33

M.A. Wimmer (Ed.): KMGov 2003, LNAI 2645, pp. 163-174, 2003.

(C) Springer-Verlag Berlin Heidelberg 2003 
nal sources like the Internet. On the other hand, tacit knowledge of authors of the documents' provides important context to them, which cannot be effectively intercepted.

Knowledge management [12] generally deals with several activities relevant in knowledge life cycle [1]: identification, acquisition, development, dissemination (sharing), use and preservation of organization's knowledge. Our approach to knowledge management in the e-Government context supports most of the activities mentioned above. Based on this approach, a Web-based system Webocra [1] has been designed and implemented. It is being now tested on pilot applications at Wolverhampton (UK) and in Kosice (Slovakia). Firstly, it provides tools for capturing and updating of tacit knowledge connected with particular explicit knowledge inside documents. This is possible due to ontology model, which is used for representation of organization's domain knowledge. Ontology with syntax and semantic rules provides the 'language' by which Webocrat(-like) system can interact at the knowledge level [8].

Use of ontology enables to define concepts and relations representing knowledge about a particular document in domain specific terms. In order to express the contents of a document explicitly, it is necessary to create links between the document and relevant parts of a domain model, i.e. links to those elements of the domain model, which are relevant to the contents of the document. Model elements can be also used for intelligent search and retrieval of relevant documents.

Existence of a knowledge model (ontology) in the center of the system is the key difference to approaches followed by other $5^{\text {th }}$ FP projects like EDEN (they are strong in use of natural language processing techniques supporting communication between citizens and public administrations) or DEMOS (very elaborated approach focused on on-line consultation).

The rest of this paper is organized as follows. Sect. 2 describes the functional overview of the Webocrat system, following with Sect. 3 describing its architecture. Sect. 4 presents three from the knowledge management point of view interesting aspects of the Webocrat system. The first one is example of ontology for a specific eGovernment application. The second one is an example of a new service required by users, offered using Webocrat in the first trial and its knowledge enhanced extension for the second trial. Finally, knowledge supported personalization offered by the Webocrat system is described in greater details. Sect. 5 makes a brief summary of the paper.

\section{Webocrat System Functional Overview}

From the point of view of functionality of the WEBOCRAT system it is possible to break down the system into several parts and/or modules [11]. They can be represented in a layered sandwich-like structure, which is depicted in Fig. 1.

1 EC funded project IST-1999-20364 Webocracy (Web Technologies Supporting Direct Participation in Democratic Processes) 


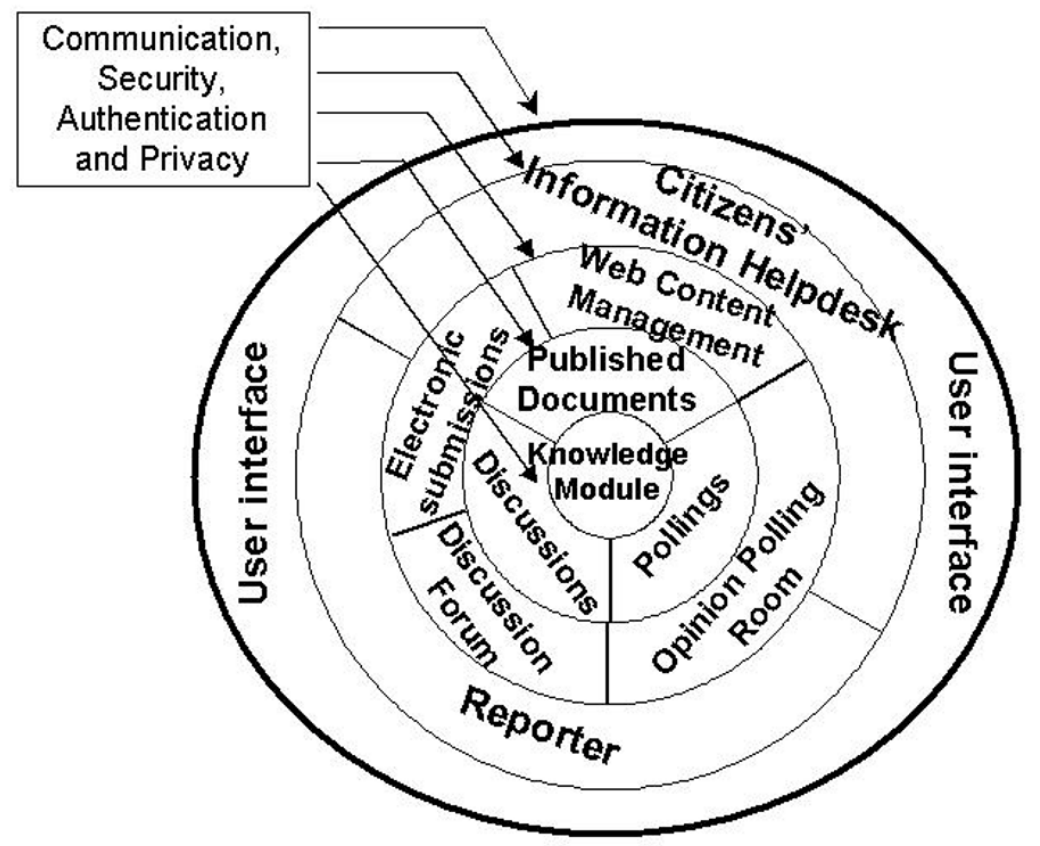

Fig. 1. WEBOCRAT system structure from the system's functionality point of view

\subsection{First Layer}

A Knowledge Model module occupies the central part of this structure. This system component contains a conceptual model of a domain. The purpose of this component is to index all information stored in the system in order to describe the context of this information (in terms of domain specific concepts). The central position symbolizes that the knowledge model is the core (heart) of the system - all parts of the system use this module in order to deal with information stored in the system (both for organizing this information and accessing it).

\subsection{Second Layer}

Information stored within the system has the form of documents of different types. Since three main document types will be processed by the system, the document space can be divided into three subspaces - publishing space, discussion space, and opinion polling space. These areas contain published documents, users' contributions to discussions on different topics of interest, and records of users' opinions about different issues, respectively. 


\subsection{Third Layer}

Since each document subspace expects different way of manipulating with documents, three system's modules are dedicated to them. Web Content Management module (WCM) offers means to manage the publishing space. It enables to prepare documents in order to be published (e.g. to link them to elements of a domain model), to publish them, and to access them after they are published. Discussion space is managed by Discussion Forum module (DF). The module enables users to contribute to discussions they are interested in and/or to read contributions submitted by other users. Electronic Submissions module (ES) enables users to access special part of the document space comprising their formal or informal submissions to local authority. Opinion Polling Room module (OPR) represents a tool for performing opinion polling on different topics. Users can express their opinions in the form of polling.

\subsection{Fourth Layer}

In order to navigate among information stored in the system in an easy and effective way, this layer is focused on retrieving relevant information from the system in various ways. Two modules represent it, each enabling easy access to the stored information in a different way. Citizens' Information Helpdesk module $(\mathrm{CIH})$ is dedicated to search. It represents a search engine based on the indexing and linking (to knowledge model) of stored documents. Its purpose is to find all those documents, which match user's requirements expressed in the form of a query defined by means of a free text, or a query composed from concepts from domain model, or by document attributes (like author, date of issue etc.).

The other module performing information retrieval is the Reporter module (REP). This module is dedicated to providing information of two types. The first type represents information in an aggregated form. It enables to define and generate different reports concerning information stored in the system. The other type is focused on providing particular documents - but unlike the $\mathrm{CIH}$ module it is oriented on off-line mode of operation. It monitors content of the document space on behalf of the user and if information the user may be interested in appears in the system, it sends an alert to him/her.

\subsection{Fifth Layer}

The upper layer of the presented functional structure of the system is represented by a user interface. It integrates functionality of all the modules accessible to a particular user into one coherent portal to the system and provides access to all functions of the system in a uniform way. In order for the system to be able to provide required functionality in a real setting, several security issues must be solved. This is the aim of the Communication, Security, Authentication and Privacy module (CSAP) [4].

Technical achievements comprise also a system designed to provide automatic routing of messages from citizens to the appropriate person within the public admini- 
stration (ES module); tools for easy access to public administration information and to competitive tendering (WCM module) and personalization support (REP modules) that will be described in greater details in Sect. 4 .

\section{Webocrat System Coarse Architecture}

Webocrat system has client-server architecture [7]. This is illustrated by a top-level system structure diagram, which is depicted in Fig. 2.

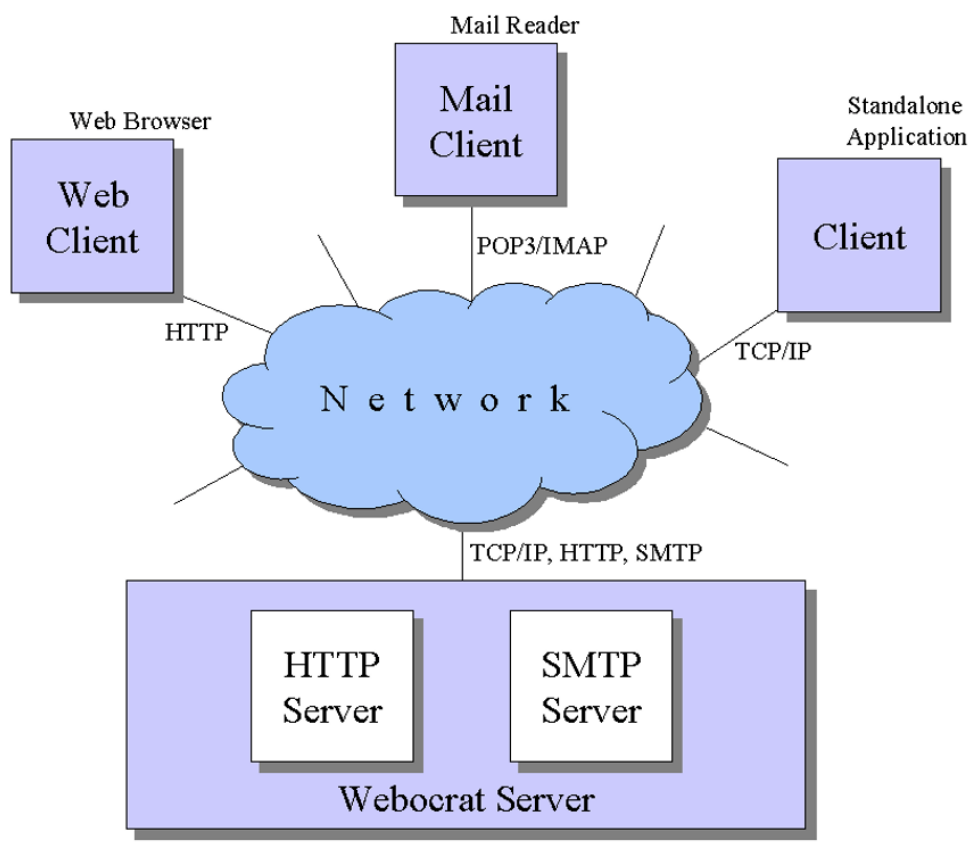

Fig. 2. Top-level decomposition of the Webocrat system structure

Three types of client software are expected in the Webocrat system - Web based clients, mail readers, and standalone applications (for the sake of simplicity they are called just clients). Mail clients are specialized in supporting of user alerting on events that could be of interest for these users as well as sending of replies to their electronic submissions. In order to provide users with mail messages, no special requirements are defined for mail clients - common mail user agents are perfectly usable.

Standalone clients represent specialised clients that are not intended for many users. Each of these clients focuses on a small set of functions provided by the system. Users working with these clients take special positions in maintaining the system and the information stored within it. At this stage of system design, three standalone clients are considered - ontology editor for building and maintaining a knowledge model, 
annotator for enriching documents, which should be uploaded on the Webocrat server by a set of links to elements from the domain model, and administrator tool for administering the whole system.

In addition to that, this type of client can represent an interface to an existing information system (i.e. the client is used by some software and not by people). For example, some external system can submit documents which should be published in the publishing space, together with information which (type of) users can access these documents, to what elements of the domain model these documents should be linked before their publication, etc.

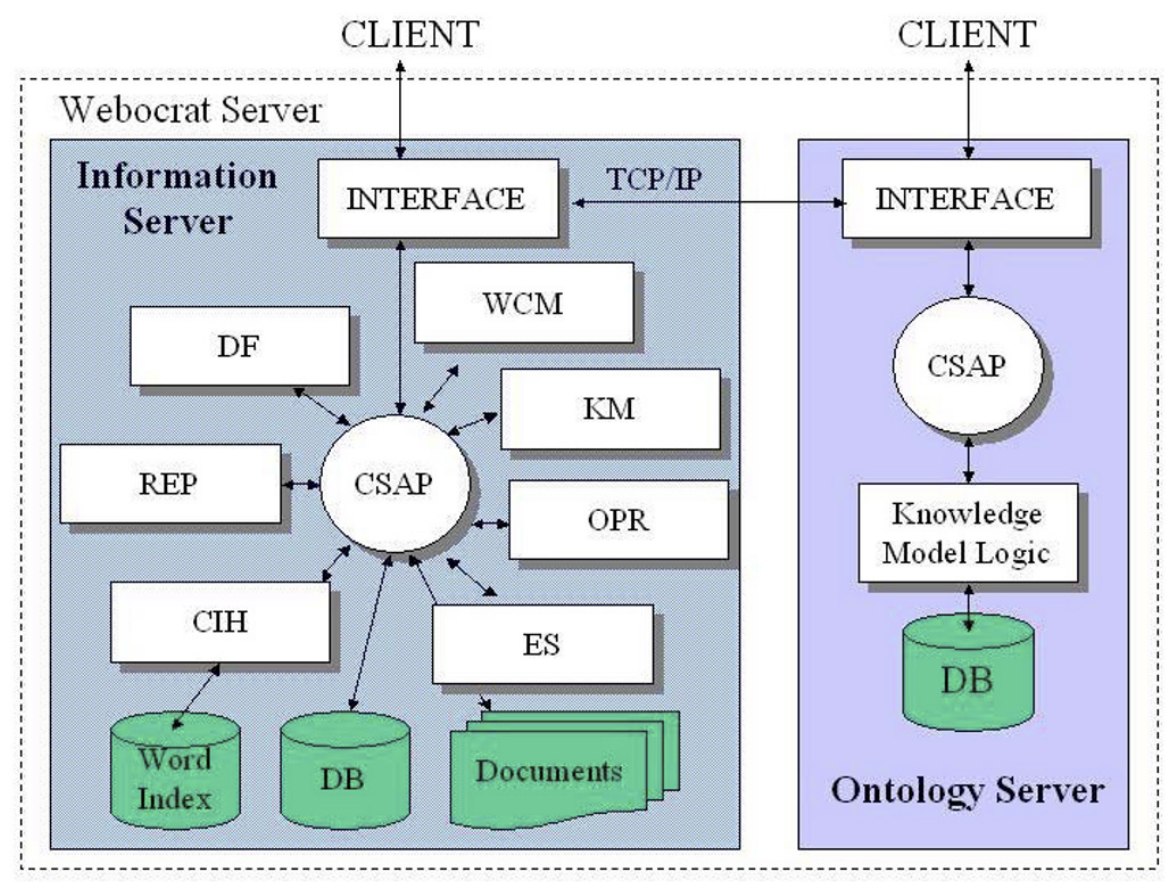

Fig. 3. Coarse grain structure of the Webocrat server

The most common type of client is a web client. The Webocrat clients are either a standard Web browser or any software, which uses the HTTP protocol to communicate with the Webocrat system. Web client is dedicated for main functions offered by the system - accessing information published in the publishing space, sharing ideas and opinions in various discussions, accessing of users' electronic submissions (e.g. a legacy system used at the local authority for recording and managing of the mail correspondence) and providing opinions on different issues by participating in some opinion polling. This type of client targets wide audience of users.

The Webocrat server itself is composed of several modules. The structure of the server is depicted in Fig. 3. 
As discussed above, the Webocrat system consists of several modules that can be installed. Basically, these modules can be divided into two relatively separate parts splitting the Webocrat server into two separate servers - Webocrat Information Server and Webocrat Ontology Server. Since these servers can be installed on separate machines, which could be far away from each other (this covers also the possibility of their installation on the same machine), the communication between them is based on the TCP/IP protocol family.

This division of responsibilities for domain models and for documents containing information of interest for users enables easier maintenance of both domain models and documents and better utilization of available resources. As an example of this can serve a case where one ontology server is shared by several information servers (using the same domain model or working with different models). Another example, an extreme one, is not to use any ontology at all and relying only on full-text retrieval of documents - in this case there is no need to install the ontology server.

\section{Pilot Applications}

Within this project, a special attention is paid to the process of integrating WEBOCRAT tools into practical applications. Rather than separating phases of development followed by the deployment of pilot applications, the process of WEBOCRAT tool and methodology development is intertwined with deployment from early beginning (all pilot applications are splitted into two trial phases). The project started with detailed analysis of user requirements (result of this study has been published in [10]). Early deployment provides important feedback and drives their further development.

The Webocracy project uses a case centered and user-driven approach. The pilot applications provide the context for all the implementation and evaluation activities. Each case provides a specific test-bed for the technology and methodology enabling on-line (Web-based) support to services and operation of public administrations. Each case is configured according to the needs and "public context" of the particular user partner.

The first trials have been devoted to basic features of DF, WCM and OPR modules [2]. The second trials will involve all features on knowledge modeling technology, alerting and personalization.

In each phase, each of the user partners conducts trials concurrently. Wolverhampton City Council (WCC) is responsible for the design, specification and evaluation of the trials in the UK. The Local Authority Kosice - City ward Tahanovce (LATA) and The Local Authority Kosice - City ward Dargovskych hrdinov (LAFU) are responsible for pilot applications and trials in Slovak Republic.

The first trial in WCC did make use of DF and OPR modules, and involved partner agencies and citizens. First trial in LATA used WCM and OPR modules. LATA focused their pilot application on web publishing and content management. In LAFU the trial was focused on ES module [2]. 


\subsection{Example of a Knowledge Model}

The preliminary model for the pilot WCC's pilot application [6] identifies five main classes:

Locations are entities with a geographical location, and are either areas (such as the area served by a local authority, or the ward which elects a local representative), or premises (such as office buildings, schools or hospitals). Slots are used to describe the geographical relationship between locations (a school is located in a ward, a ward is part of a local authority area etc.).

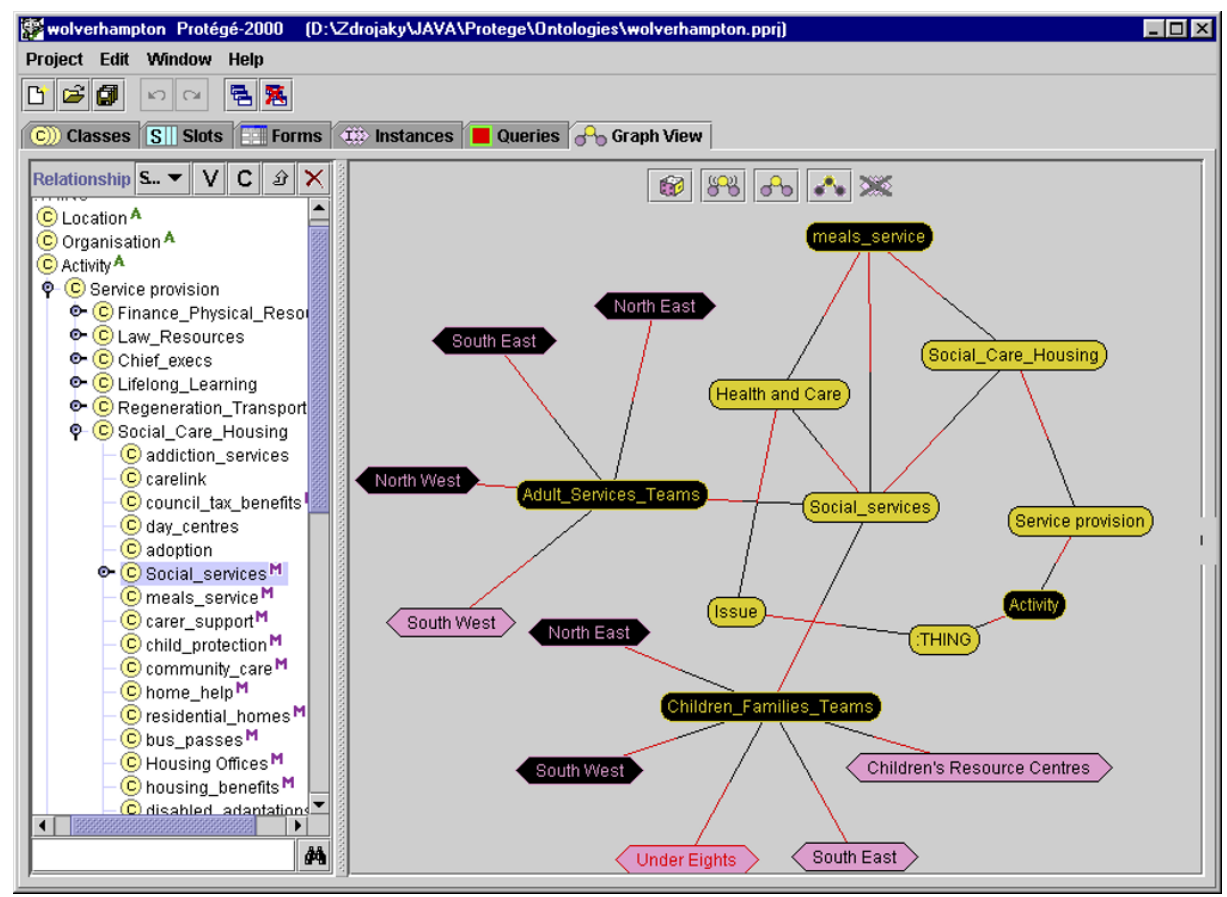

Fig. 4. Coarse grain structure of the Webocrat server

Organisations are the various public agencies, community groups and businesses that have a role in the city, and their departments or sections. This class also includes the various partnerships between these individual organisations. Slots are used to describe the hierarchy of organisation structures, and the relationships to areas served and premises occupied.

Activities describe either the provision of a service by an organisation (see Fig. 4), or the contribution of an organisation to a partnership. Slots are used to establish these relationships to organisations.

Issues are broad topics, which may be raised in discussion and consultation with citizens. These may not relate in a simple way to the structures or services of organisa- 
tions. Slots will be used to identify the links between issues, services and organisations.

Persons are individual contacts - either officers or elected representatives. Slots are used to relate them to the activities for which they are the appropriate contact. Elected representatives are also linked via slots to the areas that they are representing.

\subsection{Example of a New Service}

Here presented service has been implemented as a result of one special requirement raised by LAFU within the user requirements analysis carried on at the beginning of the project [10].

At present state at LAFU and LATA no citizen can check on the processing status of his/her document(s) submitted to the Local Authority. User knows just the processing deadline, which is 30 days after document entry date. The proposed WEBOCRAT pilot application should enable the user to check the processing status of his/her submitted document(s). WEBOCRAT system will enable easy electronic submission of documents to the Local Authority. (In second trial after positive authorization, in first as a anonymous user) any citizen will be able to see how particular submissions have been handled.

Analysis of first trial in LAFU indicates possible decrease in overhead expenses as a consequence of lower communication costs but only by a negligible amount. It also indicates that the WEBOCRAT system will simplify the work at LA. It is worth noting that the main aim of this trial was to provide citizens with a new type of service, not to decrease significantly expenses of LA.

Because of the success of the new services offered within the first trial, LAFU decided to continue in first trial and the functionality of the system will be continuously extended as new Webocrat modules will be added up to the second trial.

The most successful was in LAFU's opinion during the first trial the CM module with the possibility to submit a request/complaint electronically and even tracking the processing status of submitted document. Therefore, also within the second trial the main focus will remain on this service, enhancing it significantly using functionality of the whole Webocrat system.

Current WEBOCRAT pilot application enables users to submit a complaint/suggestion electronically and check the processing status of the submitted document(s). No authorization is provided yet. Therefore all users can see all submitted documents as well as their processing status. For the second trial, authorization and fully personalized access will be provided. But the user will have a free choice whether his/her complaint/suggestion should be accessible form him/her only, or for other users as well.

Moreover, a number of standard forms will be provided using CM module and will enable citizens to submit them electronically using Webocrat system. The system will automatically route the form to the LA employee responsible for handling it. This should lead to a significant time saving when compared to the current practice, where all mails are coming first to a specialized person for that registering them and for- 
warding to the superintendent of the LA, who distributes the mails to appropriate departments, more specifically to head of departments. Head of department assigns it further to a particular employee for processing. WEBOCRAT system application by means of the ES module will lead to a reengineering of this administration process saving significant amount of time and effort.

In order to support intelligent retrieval ( $\mathrm{CIH}$ module) within all electronic submissions, as well as various types of analysis (REP module), the following links to the conceptual knowledge model should be created for each electronic submission (document).

A link to department that will be responsible for its processing,

A link to a clerk, who was assigned responsible for processing this matter,

A link explaining type of the given electronic document (e.g. a special form, request, application etc.),

A link explaining topics deals by this document (for informal submissions only).

For submission of pre-defined forms the Webocrat system will do all the linking automatically. For informal matters employees (with Webocrat system support) may do it.

Statistical analysis of documents submitted to local authority will be supported by the REP module functionality. Based on the links to knowledge model it will be possible to analyze e.g. distribution of citizens' submissions into different topics resulting in information about what areas are problematic or overloaded. The other possibility is to analyze efficiency of processing electronic submissions by particular employees, departments, etc.

\subsection{Knowledge Based Personalisation}

Since the system can contain a lot of information in different formats (published information, discussion contributions, etc.), it may not be easy to find exactly the information user is looking for. Therefore he/she has the possibility to create his/her profile in which he/she can define his/her interests and/or preferred way of interacting with the system.

When defining an area of interest, user selects elements from a domain model (or subparts of this model). In this way user declares that he/she is interested in topics defined by the selected part of the domain model.

The definition of user's area of interest enables alerting - user can be alerted, e.g. on changes of the domain model, a new opinion polling, or publishing of a new document, opening of a new discussion, etc. User has the possibility to set alerting policy in detail on which kind of information he/she wants to be alerted in what way (including extreme settings for no alerting or alerting on each event taking place in the system). The system compares each event (e.g. submission of a discussion contribution, publishing a document, etc.) to users' profiles. If result of this comparison is positive, i.e. the user may be interested in the event, then the user is alerted.

Alerting can have two basic forms. The first alternative is represented with notification using e-mail services. User can be notified on event-per-event basis, i.e. he/she 
receives an e-mail message for each event he/she is alerted on. Alternatively, it is possible to use an e-mail digest format - user receives e-mail message, which informs him/her about several events. The way of packaging several alerts into one e-mail message depends on user's setting. Basically, it can be based on time intervals and/or the size of e-mail messages.

The other alternative is a 'personal newsletter'. This does not disturb user at unpredictable time - user simply can access his/her newsletter when he/she desires to be informed what is on in the system. Moreover, he/she can access it from arbitrary gadget connected to the Internet. The personal newsletter has the form of a document published in the publishing space. This document is generated by the system and contains links to all those documents, which may be of interest for the user. Since the document is generated when user logs in, it can cover all information submitted and/or published since the last user's visit.

User registered in the system as an individual entity (i.e. not anonymous user) is provided with a personal access page ensuring him/her an individual access to the system. This page is built in an automatic way and can consist of several parts. Some of them can be general and the others are user-specific.

The former can serve as a starting point for browsing all published documents accessible to the user, all discussions he/she is allowed to participate in (in passive or active way), all running polls for which he/she is eligible, using search facilities of the system, read hot information, etc. The latter parts are devoted to user's personal newsletter, links to documents and discussions topics of which match the user's area of interest.

User can use his/her personal access page in an active way. For example he/she can make notes, which documents from those offered by the system he/she intends to read and/or in which he/she is not interested. Or he/she can store links to those documents he/she has found exceptionally interesting, or to which he/she would like to return later.

\section{Conclusions}

In this paper, a system called Webocrat has been presented as an attempt to shift eGovernment portals toward a customized assistance and knowledge enhanced services. The Webocrat system applies a knowledge-based approach. The functional overview, as well as basic architecture of the system has been presented.

Webocrat approach is user-centered, focusing on pilot applications that are divided into two trials. At the time of writing the first trials, beta version of the full Webocrat system has been available for testing and the second trials have just started (scheduled for March - May 2003). First information about the real experience with full Webocrat system could therefore be provided at the time of the conference.

In this paper, three examples of the Webocrat potential from the knowledge management perspective have been described. 
Acknowledgements. We would like to thank our project partners for helpful comments and stimulating discussions. This work is done within the Webocracy project, which is supported by European Commission DG INFSO under the IST program, contract No. IST-1999-20364 and within the VEGA project 1/8131/01 "Knowledge Technologies for Information Acquisition and Retrieval" of Scientific Grant Agency of Ministry of Education of the Slovak Republic.

The content of this publication is the sole responsibility of the authors, and in no way represents the view of the European Commission or its services.

\section{References}

1. Abecker A., Bernardi A. Hinkelmann K. Kühn, O. \& Sintek M.: Toward a Technology for Organizational Memories, IEEE Intelligent Systems, 13 (1998) 40-48

2. Bocock, R., Cizmarik, T., Novacek E., Paralic J. and Thomson, P.: Specification of pilot applications and design of trials. Webocracy Technical Report R12.1, Technical University of Košice (2001)

3. Borghoff U. M. \& Pareschi R. (Eds.): Information Technology for Knowledge Management. Springer Verlag (1998)

4. Dridi, F. and Pernul, G. and Unger, V.: Security for the electronic government. In Proceedings of the European Conference on E-Government, Trinity College, Dublin, Ireland (2001) 99-110

5. Dzbor M., Paralic J. and Paralic, M.: Knowledge management in a distributed organization. In: Proc. of the $4^{\text {th }}$ IEEE/IFIP International Conference BASYS'2000, Kluwer Academic Publishers, London (2000) 339-348

6. Macej P., Novacek E., Thomson P., Paralic J. and Cizmarik T.: Conceptual Knowledge Models (Phase I). Webocracy Technical Report R12.2.1, Technical University of Košice (2002)

7. Mach M., Sabol T., Dridi F., Palola I., Furdik K., Thomson P., Cizmarik T., Novacek E.: Edited annual report for publication. Webocracy Technical Report R1.1.3, Technical University of Košice (2001)

8. Newell A.: The Knowledge Level. Artificial Intelligence 18 (1982) 87-127

9. Nonaka I., Takeuchi H.: The Knowledge Creating Company: How Japanese Companies Create the Dynamics of Innovation. Oxford Univ. Press (1995)

10. Paralic, J., Sabol, T.: Implementation of e-Government Using Knowledge-Based System. In. Proc. of the $12^{\text {th }}$ Int. Workshop on Database and Expert Systems Applications, (W06: 2nd Int. Workshop on Electronic Government), Munich (2001) 364-368

11. Paralic, J., Sabol, T., Mach, M.: A System to support E-Democracy. Proc. of the First International Conference EGOV 2002, Aix-en-Provence, France, LNCS 2456, Electronic Government, R. Traunmuller, K. Lenk (Eds.), Springer Verlag (2002)

12. Tiwana A.: The Knowledge Management Toolkit. Prentice Hall (2000)

13. Traunmüller, R. \& Wimmer, M.: Directions in E-Government: Processes, Portals, Knowledge. Proc. of the Int. Workshop "On the Way to Electronic Government" in Conjunction with DEXA (Munich, Germany), IEEE Computer Society Press, Los Alamitos, CA (2001) 313-317 